\title{
Incidence of Tuberculosis as a Opportunistic Infection in Patients with HIV Getting Combined Anti Retroviral Therapy
}

\section{Manoj Koirala ${ }^{1}$, Buddhi Bahadur Thapa ${ }^{1}$,Basanta Kumar Tamrakar ${ }^{1}$,Surya Bahadur Hamal ${ }^{1}$, Bishow Raj Baral ${ }^{1}$, Buddhisagar Lamichanne ${ }^{1}$, Anand Nepal ${ }^{2}$, Aarati Acharya ${ }^{3}$, Roshan Pangeni ${ }^{4}$}

${ }^{1}$ Department of Internal Medicine, Pokhara Academy of Health Sciences, Western Regional Hospital.

${ }^{2}$ Department of Dermato-Venerology, Pokhara Academy of Health Sciences, Western Regional Hospital.

${ }^{3}$ Charak Academy Pvt.Ltd., Nursing department.

${ }^{4}$ Department of Radiology, Pokhara Academy of Health Sciences, Western Regional Hospital.

\section{Correspondance:}

Dr. Manoj Koirala (MD)

Pokhara Academy of Health Sciences

Western Regional Hospital, Pokhara, Nepal

Email: koiralamanoj34@hotmail.com

Article received : $15^{\text {th }}$ May, 2018

Article accepted : $12^{\text {th }}$ August, 2019

\section{ABSTRACT}

Introduction The risk of active Tuberculosis increases after HIV seroconversion. It progressively increases with declining immunity. Tuberculosis (TB) epidemics in HIV has led to a dramatic upsurge in global TB incidence, resulting in remarkable increase in morbidity and mortality. Many clinical studies had shown that TB is one of the commonest opportunistic infection. This study is conducted to see the incidence of tuberculosis as an opportunistic infection in patients with HIV getting combined antiretroviral therapy (cART) Materials and Methods: Thiswasahospitalbasedcrosssectional comparative study. Five hundred patients with HIV who are getting cART were enrolled in the study. Results: Analysis of individuals with HIV in our study amongst the 500 patients 266 (53.2\%) were males and the remaining 234 (46.68\%) were females. Amongst these HIV patients 48(18.04\%) males and $17(7.26 \%)$ females had tuberculosis. 65(13\%) of patients were TB/HIV co-infected. $2(4.1 \%)$ of these male TB/HIV patients had cervical gland TB. Conclusion: The incidence of tuberculosis in HIV infected individuals is high. TB is the commonest opportunistic infection (OIs) in people living with HIV/AIDS. Males are predominant gender to have TB/HIV co-infection. KeyWords: Combination antiretroviral therapy (cART),Human immunodeficiency virus(HIV), Opportunistic infections(OIs), Tuberculosis(TB). 


\section{INTRODUCTION}

Tuberculosis (TB) and Human immunodeficiency virus (HIV) constitute the main burden of infectious disease in poor resource-limited countries like Nepal. World Health Organization (WHO) estimate that there are more than 9 million new active cases of TB and close to 2 million deaths per year. 2.6 million new cases of HIV infection and 1.8 million AIDS-related deaths occur per year. ${ }^{1,2}$ Particularly TB HIV co-infection has diagnostic and therapeutic challenges. It exerts extreme pressure on health care systems in Asian countries with large numbers of co-infected individuals.

TB and HIV potentiate each other and accelerate the deterioration of immunological functions resulting in premature death if untreated. Around 14 million individuals are estimated to be dually infected worldwide. TB is the commonest single cause of death in HIV/AIDS individuals and accounting for approximately $26 \%$ of AIDS-related death, $99 \%$ of which occur in developing countries ${ }^{3-5}$.

Both TB and HIV have profound effects on the immune system, as they are capable of disarming the host's immune responses through mechanisms that are not fully understood. HIV co-infection is the most powerful known risk factor for progression of $\mathrm{M}$. tuberculosis infection to active disease, increasing the risk of latent TB reactivation 20 -fold ${ }^{4,6}$. Similarly, TB has been reported to exacerbate HIV infection ${ }^{7,8}$. Various evidences have shown that inborn errors of immunity and genetic polymorphisms both have an impact on susceptibility to TB and HIV ${ }^{9}$.

Poverty, HIV, and drug resistance are major contributors to the resurging global TB epidemic . ${ }^{5,6}$ Approximately ninety-five (95\%) percent of TB cases occur in developing countries. Approximately 1 in 14 new TB cases occur in individuals who are infected with HIV 85 percent of these cases occur in Africa. ${ }^{4}$ An estimated half million cases of multidrug resistant (MDR)-TB also occur annually.
Socioeconomic development, access and quality of health services appear to be at least as important as any specific TB control measure. Risk factors for TB may be divided into issues related to host immunity (e.g, immunologic defects that lead to increased susceptibility to infection) and issues related to environmental exposure to infection.

The rate of TB is higher among men than women, throughout life. This is a longstanding observation thought to reflect more frequent TB exposure in the community among men than women ${ }^{10}$.

\section{MATERIALS AND METHODOLOGY}

It is a cross sectional, retrospective, hospital based observational study. Patients attending in ART clinic, OPD or admitted in medical ward of Pokhara academy of health sciences, Western Regional Hospital were evaluated. A total of five hundred HIV seropositive patients were included in the study. Data were collected using a structured proforma covering the relevant details. Data entered by using Microsoft Excel and statistical analysis was done by SPSS software version 20. Result obtained from the study was discussed with reference to current world literature.

\section{RESULTS}

Among the 500 patients, $266(53.2 \%)$ were males and the remaining $234(46.68 \%)$ were females. Amongst these HIV patients 48(18.04\%) males and $17(7.26 \%)$ females had tuberculosis. $65(13 \%)$ of patients were TB/HIV coinfected. 2(4.1\%) of these male TB/HIV patients had cervical gland TB.

Table 1: Gender wise distribution of HIV patients:

\begin{tabular}{lll}
\hline Gender & Frequency & Percent \\
\hline Male & 266 & 53.2 \\
\hline Female & 234 & 46.8 \\
\hline Total & 500 & 100 \\
\hline
\end{tabular}


Table 2: TB/HIV coinfection amongst male and female patients:

\begin{tabular}{lccc}
\hline Gender & $\begin{array}{l}\text { HIV } \\
\text { seropositive } \\
\text { patients }\end{array}$ & $\begin{array}{l}\text { Tuberculosis } \\
\text { coinfection }\end{array}$ & Percent \\
\hline Male & 266 & 48 & $18.04 \%$ \\
\hline Female & 234 & 17 & $7.26 \%$ \\
\hline Total & 500 & 65 & $13 \%$ \\
\hline
\end{tabular}

\section{DISCUSSION}

The incidence of tuberculosis in immunocompromised individuals in their life time is approximately $5-10 \%$, but in HIV positive patients, the risk of developing active TB disease is around $50 \%$. TB occurs in all socioeconomic strata and ethnic groups but the prevalence rates have been clearly linked to poverty. Malnutrition, HIV/AIDS, active or passive smoking and diabetes are all strong risk factors for TB. Nepal is poor and economically deficient country so is endemic for tuberculosis. Thousands of HIV patients were getting HIV treatment along with tuberculosis. It is one of the commonest cause of morbidity in patients with HIV/AIDS.

Amongst the 500 seroposotive HIV/AIDS patients who are getting cART included in this study, $266(53.2 \%)$ were males and 234 (46.68\%) were females. Total $65(13 \%)$ of patients were TB/HIV coinfected. Amongst these 48(18.04\%) males and $17(7.26 \%)$ females had tuberculosis. $2(4.1 \%)$ of these male TB/HIV patients had cervical gland TB. Mitku, Aweke Abebaw et al had conducted the similar study of 571 seropositive HIV patients and found that $158(27.7 \%)$ of patients had pulmonary tuberculosis ${ }^{11}$. Amongst these female patients who had TB/HIV co-infection accounted for a larger proportion $107(69 \%)$ compared to male patients 48(31\%). These study had shown that tuberculosis is a major comorbid condition as found in our study.

Belay M. et al. had found that amongst 287 pulmonary TB suspects who were tested for HIV infection, $82(28.6 \%)$ were HIV positive. A significantly higher proportion of bacteriologically confirmed pulmonary TB patients $40(40.4 \%)$ were HIV co-infected compared with patients without bacteriological evidence for pulmonary TB 42 (22.3\%). Overall prevalence of TB HIV co-infection in the current study is high.

Vanisri H R et al had conducted the study of thirtysix HIV-positive patients with lymphadenopathy who underwent FNAC $^{13}$. Mycobacterium tuberculosis was found in $20(58.3 \%)$ cases. Cervical lymph node was the most common site. Majority of the patients were males. The maximum number of cases had tuberculosis $(58.3 \%) .2(4.1 \%)$ of these male TB/HIV patients had cervical gland TB in our study but we had included all patients with HIV/AIDS regardless of lymphadenopathy.

Khosravi AD et al had processed 50 samples including 45 sputum (90\%), 3 pleural fluid (6\%) and 2 CSF (4\%). ${ }^{14} 8$ were sputum culture positive $(16 \%)$, 7 were positive for AFB stain (14\%) and 13 were PCR positive (26\%). All the positive results were in sputum samples which strongly related to the pulmonary tuberculosis. The majority of infections were with M. tuberculosis (18\%) and this shows the importance of this mycobacterial co-infection in HIV positive patients as compared to our study.

Ashwin S et al. studied the prevalence of TB among 287 sex trafficked Nepalese girls and women in whom a high HIV prevalence was previously reported. ${ }^{15}$ They Investigated with sputum smear for acid-fast bacilli, radiographs, histories of medical tests or case records. There were 17 cases of TB that developed after rescue. The majority of cases $(70 \%)$ were likely pulmonary TB. Nearly 9 in 10 individuals who developed TB were HIV co-infected. This result showed high prevalence of HIV and TB co-infections and is comparable to our study as TB is the commonest OIs in HIV seropositive individuals.

Manisha Ghate et.al found Tuberculosis was the most common OI with an incidence of 15.4 per 100 person-years in HIV infected individuals. ${ }^{16}$ The high incidence of commonly reported OIs in Indian HIV-infected individuals indicates that the emphasis needs to be given to the early diagnosis and management of tuberculosis in HIV-infected 
individuals. In our study total $65(13 \%)$ of patients were TB/HIV coinfected so result of these studies were comparable.

Similarly Esmael A et.al found the prevalence of TB-HIV co-infection was $44 \%$ (321/717). ${ }^{17}$ The prevalence of TB-HIV co-infection was high.

\section{CONCLUSION}

The incidence of tuberculosis in HIV infected individuals is high. TB is the commonest OI in people living with HIV/AIDS. Males are predominant gender to have TB/HIV co-infection. Emphasis should be given to early detection, isolation and proper treatment of HIV infection in community because TB/HIV is a rising public health problem.

\section{REFERENCES}

1. World Health Organization. Global tuberculosis control 2010. 2010.

2. UNAIDS. Chapter 2: epidemic update. UNAIDS report on the global AIDS epidemic 2010. 2010.

3. Corbett EL WC, Walker N, Maher D, Williams BG, et al. The growing burden of tuberculosis: global trends and interactions with the HIV epidemic. Arch Intern Med 2003;163:1009-21.

4. Getahun H GC, Granich R, Nunn P. HIV infection-associated tuberculosis: the epidemiology and the response. Clin Infect Dis. 2010;50(Suppl 3):S201-S7.

5. Collins KR Q-MM, Toossi Z, Arts EJ. Impact of tuberculosis on HIV-1 replication, diversity, and disease progression. AIDS Rev. 2002;4:165-76.

6. Selwyn PA HD, Lewis VA, Schoenbaum EE, Vermund $\mathrm{SH}$, et al. . A prospective study of the risk of tuberculosis among intravenous drug users with human immunodeficiency virus infection. N Engl J Med. 1989;320:54550.

7. Whalen C HC, Hom D, Lahart C, Simberkoff $\mathrm{M}$, et al. Accelerated course of human immunodeficiency virus infection after tuberculosis. Am J Respir Crit Care Med. 1995;151:129-35.

8. Modjarrad K VS. Effect of treating coinfections on HIV-1 viral load: a systematic
9. Moller M HE. Current findings, challenges and novel approaches in human genetic susceptibility to tuberculosis. Tuberculosis (Edinb) 2010;90:71-83.

10. GW. C. Epidemiology of tuberculosis. . Am Rev Respir Dis. 1982;125:8-15.

11. Mitku AA, Dessie, Z. G., Muluneh, E. K., \& Workie, D. L. . Prevalence and associated factors of TB/HIV co-infection among HIV Infected patients in Amhara region, Ethiopia. African Health Sciences. 2016;16(2):588-95.

12. Belay $M$ BG, Abebe $F$ Prevalence of tuberculosis, HIV, and TB-HIV coinfection among pulmonary tuberculosis suspects in a predominantly pastoralist area, northeast Ethiopia. . Glob Health Action. 2015;v8: 27949.

13. Vanisri H R NNM, Sunila R. Fine-needle aspiration cytology findings in human immunodeficiency virus lymphadenopathy. . Indian J Pathol Microbiol. 2008;51:481-4.

14. AD Khosravi SA, M Hashemzade, E Abasi, $\mathrm{S}$ Seghatoleslami. The relative frequency of Mycobacterium tuberculosis and Mycobacterium avium infections in HIV positive patients, Ahvaz, Iran. Asian Pacific Journal of Tropical Medicine. 2012;5:71-4.

15. Ashwin S. Dharmadhikari JG, Michele R. Decker, Anita Raj, Jay G. Silverman. Tuberculosis and HIV: a global menace exacerbated via sex trafficking. Int J Infect Dis 2009;13(5):543-6.

16. Manisha Ghate SD, Srikanth Tripathy, Madhura Nene, Preeti Gedam, Sheela Godbole, Madhuri Thakar, Arun Risbud, Robert Bollinger, Sanjay Mehendale. Incidence of common opportunistic infections in HIV-infected individuals in Pune, India: analysis by stages of immunosuppression represented by CD4 counts. International Journal of Infectious Diseases. 2009;13:e1-e8.

17. Esmael $A$ TG, Wubie $M$, Endris $M$. Tuberculosis and Human Immune Deficiency Virus Co-infection in Debre Markos Referral Hospital in Northwest Ethiopia: A Five Years Retrospective Study. J AIDS Clin Res. 2013;4(263). 